\title{
Atmospheric Transport Modeling Based Estimation of Radioactive Release from the Fukushima Dai-ichi Nuclear Power Plant Accident
}

\author{
Wolfango Plastino, Michael Schöppner, \\ Francesco Bella, Mario De Vincenzi \\ University of Roma Tre, Dept. of Physics, \\ and INFN, Section of Roma Tre \\ Rome, Italy \\ Gerhard Wotawa \\ Central Institute for Meteorology and Geodynamics \\ Vienna, Austria
}

\author{
Pavel P. Povinec \\ Comenius University, Dept. of Nuclear Physics and \\ Biophysics \\ Bratislava, Slovakia
}

\author{
Antonio Budano, Federico Ruggieri \\ INFN, Section of Roma Tre \\ Rome, Italy
}

\begin{abstract}
As a consequence of the accident at the Fukushima Dai-ichi nuclear power plant on March 2011, it is important to characterize radioactivity release into the environment. Several isotopes, amongst others caesium-137 and iodine-131, are monitored at multiple stations throughout the world by the International Monitoring System of the Comprehensive Nuclear Test Ban Treaty Organization. In this paper it is demonstrated how a worst case estimation of the radioactive release would contribute to the IMS signal. The sensitivity between source and receptor was determined using the Atmospheric Transport Modeling (ATM), running on the GRID computing facility of the Italian National Institute of Nuclear Physics (INFN) - Roma Tre. The simulations were compared with actual measurements.
\end{abstract}

Keywords-atmospheric transport modeling; caesium; iodine; nuclear power plant.

\section{INTRODUCTION}

The 2011 Tōhoku earthquake and tsunami caused severe damage to Japanese infrastructure. Especially the Fukushima Dai-ichi nuclear power plant (NPP) has been presented in the media as a threat not only to its local environment, but also as an impact to the global ecosystem. Therefore, more information on the radioactive emissions has to be gathered, but it is a difficult task to determine the actual release of radioactive material. The isotopes caesium137 and iodine-131 play a significant role here, since both are solely anthropogenic and usually only produced during nuclear weapon tests and nuclear accidents.

The Comprehensive Nuclear Test Ban Treaty Organization (CTBTO) has built up an International Monitoring System (IMS), including 80 stations to measure the atmospheric radioactivity. From these daily sampling activities the radioactive concentration $\left(\mathrm{Bq} / \mathrm{m}^{3}\right)$ of caesium137 and iodine-131 at the monitoring stations can be determined. Compared with other stations in the IMS network the station JPP38 in the city of Gunma, Japan, has continuously measured the highest concentration of both isotopes. As a second station for comparison USP79 on Hawaii, USA, has been selected.

Then Atmospheric Transport Modeling (ATM) can be used to estimate the radioactive source term at the Fukushima Dai-ichi NPP $(37.42 \mathrm{~N}, 141.03 \mathrm{E})$ that is supposed to be mainly responsible for the signal received at the stations JPP38 in Gunma $(36.31 \mathrm{~N}, 139.00 \mathrm{E})$ and USP79 $(21.52 \mathrm{~N}, 157.99 \mathrm{~W})$. The station JPP38 is in the southwest of Fukushima and with a distance of about 250 $\mathrm{km}$ it is also the closest IMS station to the assumed source, and therefore the majority of the atmospheric transport can be assumed to be over land. The second station, USP79, on the other hand, has a distance of $6,200 \mathrm{~km}$ to the assumed source, while the transport is mainly over the sea.

\section{BACKGROUND}

\section{A. Atmospheric Transport Modeling}

The relation between a source, which emits particles into the atmosphere, and the concentration at a receptor can be explained with a source-receptor sensitivity matrix. The concentration $\mathrm{c}\left(\mathrm{Bq} / \mathrm{m}^{3}\right)$ at any given receptor can be expressed as the product of a spatio-temporal source field $\mathrm{S}$ (Bq) and a corresponding source-receptor sensitivity field $\mathrm{M}$ $\left(\mathrm{m}^{-3}\right)$ at discrete locations $(\mathrm{i}, \mathrm{j})$ and time intervals $\mathrm{n}$ :

$$
c=M_{i j n} S_{i j n} \text {. }
$$

The field $\mathrm{S}$ is a multidimensional array of sources, which is transformed by the multidimensional array of multiplicators $\mathrm{M}$ into the concentration $\mathrm{c}$ that is measured at the receptor [1]. Here $M$ presents the sensitivity between source and receptor and has the dimension of $\mathrm{m}^{-3}$, whereas the inverse element of $M$ can be depicted as a dilution volume. Atmospheric Transport Modeling has been proven to be a valid tool for determining Source-Receptor Sensitivity (SRS) matrices. However, while the underlying 
calculations are naturally three-dimensional, the SRS matrix $\mathrm{M}$ is only two-dimensional. The calculations in this work have been accomplished by using the Lagrangian type model Flexpart [2]. Also the CTBTO has utilized ATM to monitor emissions from nuclear weapon tests by using Flexpart [1]. Flexpart is capable of doing forward as well as backward modeling, where both methods have advantages and disadvantages. Both, source and receptor locations, are known in the case of the Fukushima Dai-ichi accident in March 2011 and the IMS measurements at the stations JPP38 and USP79. Forward modeling is usually more efficient when the number of known sources is limited and the receptors are undefined. Backward modeling is usually more efficient when the number of receptors is limited and the sources are unknown. However, both possibilities can be used in this case to determine the sensitivity between source and receptor. In the frame of the presented results Flexpart has been used in the backward mode.

\section{B. Parallel computing}

In context with this research it is desirable to run multiple Flexpart jobs in order to calculate SRS fields for a number of different sources and receptors. Since the Flexpart source code is highly linear it is not efficient to parallelize it. Nevertheless, a user-defined number of Flexpart runs, normally carried out in series, can be sent as a job to a cluster (or grid) computer for parallel execution. Flexpart has been ported to a local cluster computer at the Grid Lab of INFN and Department of Physics of University of Roma Tre (Rome, Italy) in order to be able to do multiple parallel runs with minimum time delay [3]. The porting on the Grid Infrastructure is in progress and will allow running the program on the geographically distributed nodes of the

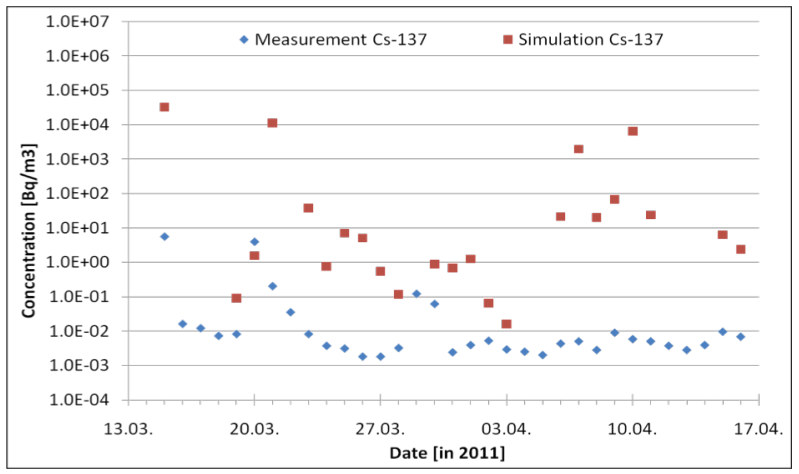

Figure 1: Comparison of concentration measurement and simulation of caesium-137 at IMS station JPP38.

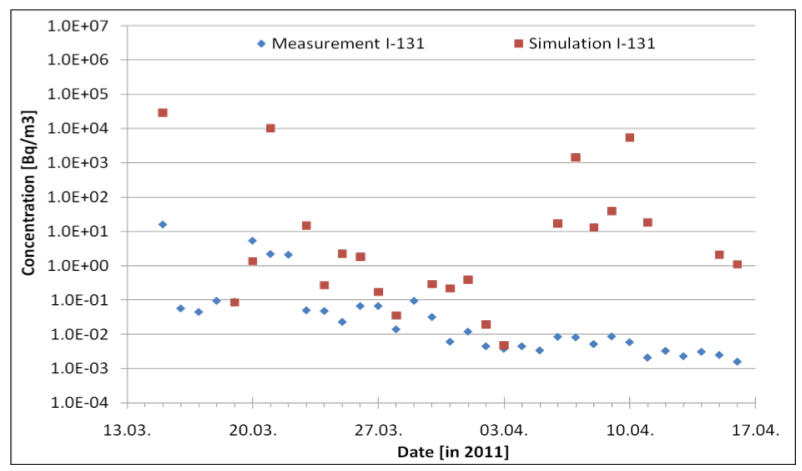

Figure 2: Comparison of concentration measurement and simulation of iodine-131 at IMS station JPP38.

\section{EUMEDGRID-Support Grid Infrastructure.}

As mentioned, the isotopes caesium-137 and iodine-131 play an important role for the estimation of the Fukushima Dai-ichi source term. The measurements of both isotopes at the IMS stations JPP38 and USP79 have been published on the website of the German Federal Office for Radiation Protection [4]. In this work the concentration measurements from 15 March 2011 until 16 April 2011 are presented and used as a basis for the following comparison with the Flexpart simulations of this worst case scenario estimation.

\section{RESULTS AND DisCUSSION}

The situation at the damaged reactors of the Fukushima Dai-ichi power plant is difficult to assess directly. Therefore, a worst case estimation of the source term is used as input data. The presented simulations assume the whole NPP as one single source, which releases radioactive particles in form of caesium-137 and iodine-131 as follows:

1. From 12 March 2011 - the day of the first explosion in a reactor building, a continuous emission of $10^{19}$ $\mathrm{Bq} /$ day per isotope has been assumed. This is a simplifying assumption that does not take into account the temporal variation caused by the fact that the number of damaged nuclear facilities increased during the first week. Three units and possibly a fuel storage pool have been damaged with different delays [5]. However, this estimated release is probably higher than a real situation at NPP.

2. Before 12 March 2011 - it has been estimated that no radioactive release took place. This assumption is significant, since releases prior to the first measurement could contribute through longer atmospheric trajectories

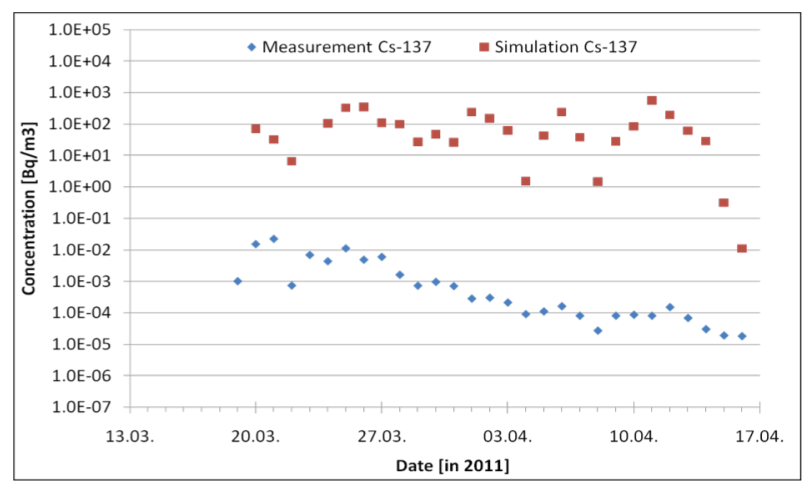

Figure 3: Comparison of concentration measurement and simulation of caesium-137 at IMS station USP79.

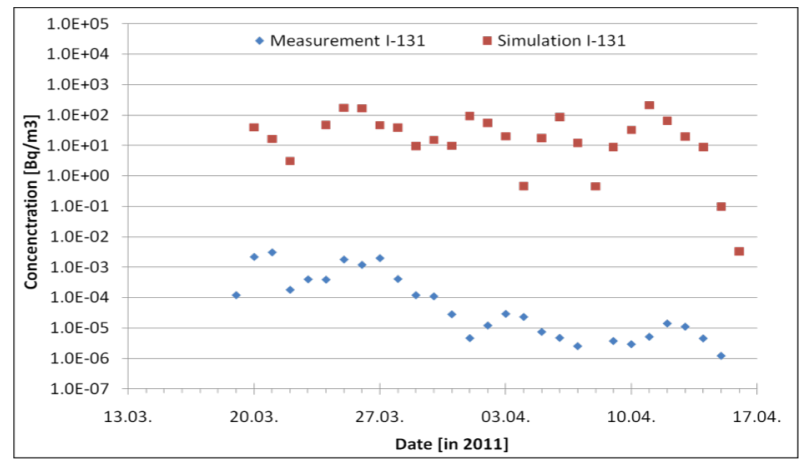

Figure 4: Comparison of concentration measurement and simulation of iodine-131 at IMS station USP79. 
to the measurement signal.

The sensitivities between the source and the receptor are calculated, including overlapping contributions from different time intervals. For the meteorological data a resolution of $1^{\circ} \times 1^{\circ}$ in terms of longitude and latitude has been used. The Flexpart simulation includes radioactive decay for both isotopes, i.e. a half-life of 30.17 years for caesium-137, and 8.02 days for iodine-131. It does not include any isotope-specific wet deposition in the atmosphere. Finally the results are summed up for each day and compared to the IMS signal. In Fig. 1 and 2 (Fig. 3 and 4) the results for caesium-137 and iodine-131 are presented and compared to the actual measurement at the IMS station JPP38 (station USP79). It is seen that the simulation depicts the common characteristics of the IMS signal.

Nevertheless, the concentrations time series is highly dependent not only from the source term, but also from the atmospheric conditions, e.g. wind direction, rain, vertical movement of air masses etc. Therefore, similar characteristics of both signals, i.e. local maxima and minima, can be explained with corresponding meteorological effects; whereas, differences in the characteristics are rather caused by a time dependent source term. However, for the station JPP38 the simulated signal is generally higher than the measured concentration, but also gives estimations in the right order of magnitude. At the USP79 station the simulation is also higher than the measurement, but in fact constantly by four to six orders of magnitude.

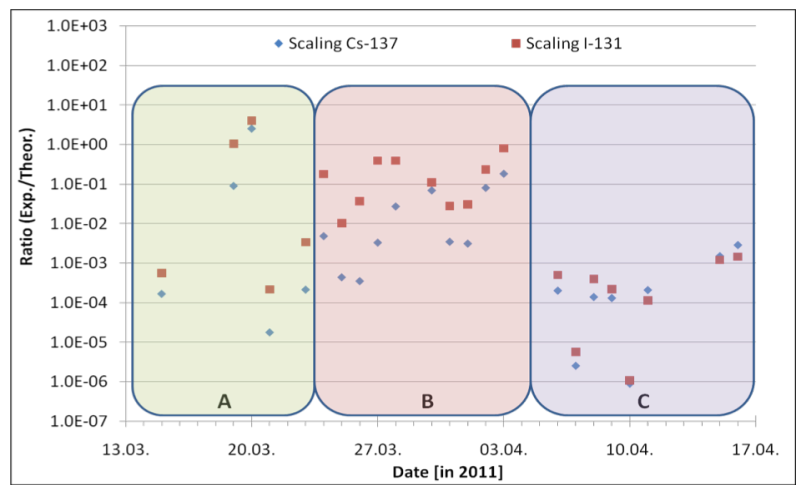

Figure 5: Scaling factors at JPP38 for caesium-137 and iodine-131, i.e. the ratios of experimental measurement and Flexpart simulation.

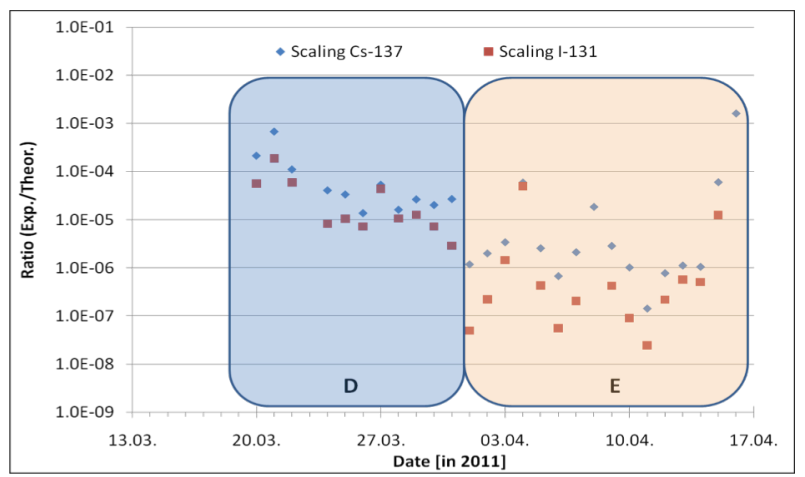

Figure 6: Scaling factors at USP79 for caesium-137 and iodine-131, i.e. the ratios of experimental measurement and Flexpart simulation.
Noted reasons for the difference are gathered in order to improve the estimation:

- due to the limited resolution of meteorological data it is clear that ATM cannot fully simulate the global atmosphere and produce straight forward results. Absolute calculations involving ATM usually have an uncertainty of one order of magnitude. It has also been discussed in recent literature [6] that local atmospheric patterns that are not resolved by the simulation can lead to altered signals;

- the conducted simulations have been done without an isotope-specific consideration of wet deposition in the atmosphere. Due to repeated rainfall in the regarded area and the regarded period a certain fraction of the radioactive particles has been washed out and not been sampled by the IMS station. It has been estimated by the author of [1] that for the JPP38 station this can lead to a signal lowered by about one order of magnitude, which would result in a one order of magnitude higher estimation of the source term. For longer trajectories, like for the USP79 station, the effect is naturally increasing;

- the basic assumption for the analysis has been a continuous and constant emission of $10^{19} \mathrm{~Bq} / \mathrm{day}$, whereas the real source term can be imagined to be strongly time dependent due to the course of events at the NPP site [5]. The increasing numbers of damaged/emitting reactors and storage pools, the explosions, and the cooling attempts have most certainly produced a time dependent source term.

As a final point of the discussion a scaling factor is introduced for both isotopes. It simply expresses the ratio of the signal measurement to the signal simulation. A value of one would mean that the signal has been estimated correctly, a value smaller (higher) than one would signify that the simulation has produced a higher (smaller) signal. However, this factor cannot be used as the correction factor for the source term, i.e. it is not equal to the ratio of the real source term to the estimated source term. This is not the case due to the possibility of atmospheric trajectories from different source times can reach the detector at the same day, and vice versa. The scaling factors for both isotopes and both stations are presented in Fig. 5 and 6. For the JPP38 station the scaling factor of iodine-131 is generally higher than of caesium-137; up to two orders of magnitude are observed, while the calculated point-to-point average suggests the factor two for the iodine-131 source term over the caesium137. For the USP79 station caesium-137 has a generally higher scaling factor, which would lead to an estimation of a stronger caesium-137 source term. However, the missing wet deposition in the simulation makes the estimation less reliable.

It is also noticeable, that the scaling factors show a time dependent behavior: for the JPP38 station three time periods can be distinguished, denoted $\mathrm{A}, \mathrm{B}$ and $\mathrm{C}$ in Fig.5. The period A shows a scattering of both scaling factors, while B (and $\mathrm{C}$ ) shows elevated (and lowered) clustering. For the 
USP79 two time periods can be distinguished: in period D both scaling factors follow a similar development, while in $\mathrm{E}$ the results are scattered. These effects are most likely due to wet deposition and a time dependent source term.

However, the time series of both scaling factors indicates a source term smaller than the estimation of a worst case scenario: from the JPP38 comparison an iodine131 source term of $10^{15}-10^{18} \mathrm{~Bq} / \mathrm{day}$ (factor 0.5 for caesium source term) is suggested. The scaling factors of the USP79 station are suggesting a much weaker iodine source term of $10^{12}-10^{15} \mathrm{~Bq} /$ day (factor 5 for caesium source term).

\section{CONCLUSION AND OUTLOOK}

Due to the severe situation in Japan, and especially at the damaged Fukushima NPP, it is difficult to directly assess the radioactive release from the Fukushima reactors. This straight-forward approach shows that through the usage of ATM the actual source term can be approximated. Based on the estimation of a worst case scenario with continuous release and the measurement at the JPP38 station, the estimation of the iodine source term can be narrowed down to $10^{15}-10^{18} \mathrm{~Bq} /$ day (factor 0.5 for caesium).

It is noticeable that an estimation of the source term based on the USP79 station would lead to an iodine source term that is smaller by three orders of magnitude.

However, the presented analysis involves only a time independent source term, which continuously emits particles. Future work will include an estimation of the source term correction factor, i.e. the adjustment of the source term in a way that the produced signal at the coordinates of JPP38 and USP79 is similar to actual measurements.

In order to accomplish a time dependent source term, a recursive usage of ATM will be necessary for step-by-step approximation. Furthermore, the inclusion of the wet deposition effect for each isotope will be added, and more IMS stations will be included in the analysis.

\section{ACKNOWLEDGEMENTS}

The authors highly acknowledge the support by National Scientific Committee Technology of INFN for the project ERMES and the European Commission under FP7 (Grant RI-246589) for the project EUMEDGRID. The authors are grateful to the Grid Lab of INFN and the Department of Physics of University of Roma Tre, and specifically to Federico Bitelli.

\section{REFERENCES}

[1] G. Wotawa, L.E De Geer, P. Denier, M. Kalinowski, H. Toivonen, R. D'Amours, F. Desiato, J.P. Issartel, M. Langer, P. Seibert, A. Frank, C. Sloan, and Y. Yamazawa, "Atmospheric transport modelling in support of CTBT verification - overview and basic concepts", Atmospheric Environment, vol. 37, pp. 2529-2537, 2003.

[2] A. Stohl, H. Sodemann, S. Eckhardt, A. Frank, P. Seibert, and G. Wotawa, "The Lagrangian particle dispersion model FLEXPART version 8.2", Norwegian Institute of Air Research, Kjeller, Norway, http://transport.nilu.no/flexpart, 2010.

[3] M. Schöppner, "Discrimination of nuclear explosions from legitimate xenon sources with atmospheric transport modeling", $\mathrm{PhD}$ Thesis at University of Roma Tre, (to be published end of 2012).

[4] German Federal Office for Radiation Protection (BfS), "Spurenanalyse deutscher Messstellen und weiterer Spurenmessstellen weltweit", http://www.bfs.de/en/ion/imis/spurenmessungen.html, April 2011.

[5] International Atomic Energy Agency, "Fukushima Nuclear Accident Update Log", http://iaea.org/newscenter/news/tsunamiupdate01.html, April 2011.

[6] W. Plastino, R. Plenteda, G. Azzari, A. Becker, P.R.J. Saey, and G. Wotawa, "Radioxenon Time Series and Meteorological Pattern Analysis for CTBT Event Categorisation“, Pure Appl. Geophys., vol. 167, pp. 559-573, 2010. 